\title{
Renormalization group analysis of the anisotropic Kardar-Parisi-Zhang equation with spatially correlated noise
}

\author{
H. Jeong ${ }^{1}$, B. Kahng ${ }^{2}$, and D. Kim ${ }^{1}$ \\ 1 Center for Theoretical Physics and Department of Physics, \\ Seoul National University, Seoul 151-742, Korea \\ ${ }^{2}$ Department of Physics, Kon-Kuk University, Seoul 133-701, Korea
}

\begin{abstract}
We analyze the anisotropic Kardar-Parisi-Zhang equation in general substrate dimensions $d^{\prime}$ with spatially correlated noise, $\langle\tilde{\eta}(\mathbf{k}, \omega)\rangle=0$ and $\left\langle\tilde{\eta}(\mathbf{k}, \omega) \tilde{\eta}\left(\mathbf{k}^{\prime}, \omega^{\prime}\right)\right\rangle=2 D(k) \delta^{d^{\prime}}\left(\mathbf{k}+\mathbf{k}^{\prime}\right) \delta\left(\omega+\omega^{\prime}\right)$ where $D(k)=D_{0}+D_{\rho} k^{-2 \rho}$, using the dynamic renormalization group (RG) method. When the signs of the nonlinear terms in parallel and perpendicular directions are opposite, a novel finite stable fixed point is found for $d^{\prime}<d_{c}^{\prime} \equiv 2+2 \rho$ within one-loop order. The roughening exponent $\alpha$ and the dynamic exponent $z$ associated with the stable fixed point are determined as $\alpha=\frac{2}{3}\left(\rho-\frac{d^{\prime}-2}{2}\right)$, and $z=2-\alpha$. For $d^{\prime}>d_{c}^{\prime}$, the RG transformations flow to the fixed point of the weak-coupling limit, so that the dynamic exponent becomes $z=2$.
\end{abstract}

PACS numbers: $61.50 . \mathrm{Cj}, 05.40 .+\mathrm{j}$ 
Recently, there have been many studies in the field of nonequilibrium surface growth. A number of discrete models and continuous equations for the surface growth phenomena have been introduced and studied [1]. One of the most interesting features in the nonequilibrium surface growth is the nontrivial dynamic scaling behavior of the interface width, i.e.,

$$
W(L, t)=\left\langle\frac{1}{L^{d^{\prime}}} \sum_{i}\left(h_{i}-\bar{h}\right)^{2}\right\rangle^{1 / 2} \sim L^{\alpha} f\left(t / L^{z}\right),
$$

where $h_{i}$ is the height of site $i$ on the substrate. $\bar{h}, L$, and $d^{\prime}$ denote the mean height, system size, and the substrate dimension, respectively. The symbol $\langle\cdots\rangle$ stands for the statistical average. The scaling function $f(x)$ approaches to a constant for $x \gg 1$, and $f(x) \sim x^{\beta}$ for $x \ll 1$ with $z=\alpha / \beta$. The exponents $\alpha, \beta$ and $z$ are called the roughness, the growth, and the dynamic exponent, respectively.

The simplest nonlinear dynamic equation describing a growing surface with lateral growth effect was introduced and studied by Kardar, Parisi, and Zhang (KPZ) [2], which is given by

$$
\frac{\partial h(\mathbf{x}, t)}{\partial t}=\nu \nabla^{2} h+\frac{\lambda}{2}(\nabla h)^{2}+\eta(\mathbf{x}, t)
$$

The noise $\eta(\mathbf{x}, t)$ is, in general, correlated spatially and temporally, $\langle\tilde{\eta}(\mathbf{k}, \omega)\rangle=0$, and

$$
\left\langle\tilde{\eta}(\mathbf{k}, \omega) \tilde{\eta}\left(\mathbf{k}^{\prime}, \omega^{\prime}\right)\right\rangle=2 D(\mathbf{k}, \omega) \delta^{d^{\prime}}\left(\mathbf{k}+\mathbf{k}^{\prime}\right) \delta\left(\omega+\omega^{\prime}\right)
$$

where $\tilde{\eta}(\mathbf{k}, \omega)$ is the Fourier transform of the noise $\eta(\mathbf{x}, t)$. When the noise is correlated only spatially, an interesting form of $D(\mathbf{k}, \omega)$ can be written as $D(k)=D_{0}+D_{\rho} k^{-2 \rho}$ [3]. Here, the $D_{0}$ term is needed to make the RG transformation closed. The white noise corresponds to the limiting case of $\rho=0$. When $\rho>0$, the noise is correlated in space with a power law. It is useful to remind that the KPZ equation is invariant under the Galilean transformation, which yields the scaling relation, $\alpha+z=2[2]$. 
Recently the anisotropic KPZ (AKPZ) equation was introduced to study the surface growth on the vicinal substrate [4]. Besides, the AKPZ equation can also be applied to various physical growth problems such as the ion-sputtered surface growth [5], and the surface growth on the reconstructed surface structure [6], etc. The AKPZ equation is written as

$$
\frac{\partial h(\mathbf{x}, t)}{\partial t}=\nu_{\perp} \nabla_{\perp}^{2} h+\nu_{\|} \nabla_{\|}^{2} h+\frac{\lambda_{\perp}}{2}\left(\nabla_{\perp} h\right)^{2}+\frac{\lambda_{\|}}{2}\left(\nabla_{\|} h\right)^{2}+\eta(\mathbf{x}, t)
$$

where $\nabla_{\perp}\left(\nabla_{\|}\right)$is the gradient along the perpendicular (parallel) directions. The anisotropy means $r_{\nu} \equiv \nu_{\|} / \nu_{\perp} \neq 1$ and $r_{\lambda} \equiv \lambda_{\|} / \lambda_{\perp} \neq 1$. Here, we consider only the case of positive $\nu_{\perp}$ and $\nu_{\|}$for stable surfaces. The AKPZ equation was studied by Wolf for the case of white noise in $2+1$ dimensions using the dynamic renormalization group ( $R G)$ method [4]. He found that when the signs of $\lambda$ 's are opposite, the nonlinear terms turn out to be irrelevant under the RG transformation. As a result, the AKPZ equation with opposite signs of $\lambda$ 's belongs to the weak coupling limit, the Edwards-Wilkinson (EW) universality class [7]. A stochastic model associated with this weak coupling behavior of the AKPZ equation in $2+1$ dimensions was introduced by the present authors [8] as a generalization of the Toom model [9]. By the simulations, we confirmed that the height fluctuations width increases logarithmically both with time before saturation, and with system size after saturation, which is the signature of the EW universality class.

In this paper, we extend the study of the AKPZ equation to the case of spatially correlated noise, $D(k)=D_{0}+D_{\rho} k^{-2 \rho}$, in general substrate dimensions $d^{\prime}$, using the dynamic RG method. Thus the previous study by Wolf corresponds to the limiting case, $\rho=0$ and $d^{\prime}=2$, of the current study. By this extension, we find that when the signs of the nonlinear coupling coefficients are opposite, a new "stable" fixed point of the RG flow exists within the one-loop order in the "off-axis region" of the parameter space $\left(U_{0}, U_{\rho}\right)$ provided $d^{\prime}<d_{c}^{\prime} \equiv 2+2 \rho$. Here $U_{0}$ and $U_{\rho}$ are dimensionless effective coupling constants, $U_{0} \equiv K_{d^{\prime}-1} D_{0} \lambda_{\perp}^{2} / \nu_{\perp}^{3}$ and $U_{\rho} \equiv K_{d^{\prime}-1} D_{\rho} \lambda_{\perp}^{2} / \nu_{\perp}^{3}$, where $K_{d^{\prime}}=S_{d^{\prime}} /(2 \pi)^{d^{\prime}}$, with $S_{d^{\prime}}$ the surface area of a unit sphere in 
$d^{\prime}$ dimensions. Moreover, the dynamic exponent $z$ associated with the fixed point is determined exactly as $z=2-\frac{2}{3}\left(\rho-\frac{d^{\prime}-2}{2}\right)$. Thus, even at $d^{\prime}=2$, the exponents $\alpha$ and $z$ are exactly obtained as $z=2-\frac{2 \rho}{3}$ and $\alpha=\frac{2 \rho}{3}$ for finite $\rho$. This result would be interesting, because it is rare to know the dynamic exponent exactly at $d^{\prime}=2$ in other surface growth problems. As the dimension $d^{\prime}$ approaches $d_{c}^{\prime}$ for finite $\rho$, the stable fixed point moves to the origin, $\left(U_{0}^{*}, U_{\rho}^{*}\right)=(0,0)$, and the nonlinear terms become irrelevant for $d^{\prime}>d_{c}^{\prime}$. Conversely, as the power $\rho$ approaches zero at $d^{\prime}=2$, the fixed point shifts towards the origin, and the weak coupling behavior derived by Wolf is recovered. On the other hand, when the signs of the nonlinear terms are the same, the behavior of the RG flows is similar to the one for the isotropic case with the correlated noise studied by Medina et al. [3], so that we will not consider this case further.

The equation we study here is the AKPZ equation, Eq. (4), in general $d^{\prime}$ dimensions, among which one dimension is assigned to the parallel dimension on the substrate, and the remaining $d^{\prime}-1$ dimensions to the perpendicular dimensions. The calculations of the RG transformation were performed by combining the two methods, one of which was introduced by Medina et al. to study the isotropic case with the correlated noise, and the other by Wolf to study the anisotropic case with the white noise. The steps of the RG transformation are following. First, the anisotropic exponent $\chi$ is introduced to relate the two characteristic length scales as $\xi_{\|} \sim \xi_{\perp}^{\chi}$, where $\xi_{\|}$and $\xi_{\perp}$ are the characteristic length scales in parallel and perpendicular directions on the substrate, respectively. Next, the coarse-graining transformation is performed within the one-loop order by integrating out the fluctuations of heights within small length scales, which correspond to the wavevectors, $e^{-l} \pi / a \leq\left|k_{\perp}\right| \leq \pi / a$ and $e^{-l \chi} \pi / a \leq\left|k_{\|}\right| \leq \pi / a$, ( $a$ is the lattice constant) for the parameters, $\nu_{\perp}, \nu_{\|}, \lambda_{\perp}, \lambda_{\|}$, and the effective coupling constants, $U_{0}$, and $U_{\rho}$. Through this coarse-graining process, the $\lambda$ 's remain unchanged, which is due to the invariance of the AKPZ equation under the Galilean transformation, 


$$
x_{i} \rightarrow x_{i}+\lambda_{i} \epsilon t, \quad h \rightarrow h+\epsilon \sum_{i} x_{i}-\sum_{i} \lambda_{i} \epsilon^{2} t / 2
$$

where $i$ denotes $\perp$ or $\|$, and $\epsilon$ is an infinitesimal angle of tilt. As a final step, the rescalings are performed as $x_{\perp} \rightarrow e^{l} x_{\perp}, x_{\|} \rightarrow e^{l \chi} x_{\|}, h \rightarrow e^{l \alpha} h, t \rightarrow e^{l z} t$. Under the rescalings, one has $\lambda_{\perp} \rightarrow e^{l(\alpha+z-2)} \lambda_{\perp}$ and $r_{\lambda} \rightarrow e^{2 l(1-\chi)} r_{\lambda}$. From the scale invariance, the scaling relation, $\alpha+z=2$, and $\chi=1$ are obtained, provided that $\lambda_{\perp} \neq 0$ and $r_{\lambda} \neq 0$. The case of $\lambda_{\perp} \neq 0$ and $r_{\lambda} \neq 0$ is much more realistic than the case that one of the $\lambda$ 's is zero. Fortunately for $\chi=1$, the recursion relations are explicitly calculable, but they could not be done for $\chi \neq 1$. We find that the $\mathrm{RG}$ recursion relations for $\chi=1$ are as follows.

$$
\begin{aligned}
& \frac{\partial \nu_{\perp}}{\partial l}=\nu_{\perp}\left[z-2-\left(U_{0}+U_{\rho}\right)\left(A-\frac{2 C}{d^{\prime}-1}\right)+U_{\rho} \frac{2 \rho B}{d^{\prime}-1}\right], \\
& \frac{\partial r_{\nu}}{\partial l}=r_{\nu}\left(U_{0}+U_{\rho}\right)\left[\left(1-\frac{r_{\lambda}}{r_{\nu}}\right) A-\frac{2 C}{d^{\prime}-1}+2 r_{\lambda} E\right]+2 \rho r_{\nu} U_{\rho}\left[\left(-\frac{B}{d^{\prime}-1}+\frac{r_{\lambda} F}{r_{\nu}}\right)\right], \\
& \frac{\partial U_{0}}{\partial l}=U_{0}\left(2-d^{\prime}\right)+U_{0}^{2}\left(G+3 A-\frac{6 C}{d^{\prime}-1}\right)+U_{0} U_{\rho}\left(3 A-\frac{6 C+6 \rho B}{d^{\prime}-1}+2 G\right)+U_{\rho}^{2} G, \\
& \frac{\partial U_{\rho}}{\partial l}=U_{\rho}\left[\left(2-d^{\prime}+2 \rho\right)+3\left(U_{0}+U_{\rho}\right)\left(A-\frac{2 C}{d^{\prime}-1}\right)-U_{\rho} \frac{6 \rho B}{d^{\prime}-1}\right] .
\end{aligned}
$$

Here $A, B, C, E, F$, and $G$ are defined as $A \equiv I\left(0,1, \frac{d^{\prime}-2}{2}, 2\right), B \equiv I\left(0,1, \frac{d^{\prime}}{2}, 2\right), C \equiv$ $I\left(0,1, \frac{d^{\prime}-2}{2}, 3\right), E \equiv I\left(2,1, \frac{d^{\prime}-2}{2}, 3\right), F \equiv I\left(2,1, \frac{d^{\prime}}{2}, 2\right)$, and $G \equiv I\left(0,2, \frac{d^{\prime}-2}{2}, 3\right)$, respectively, where

$$
I(\alpha, \beta, \gamma, \delta) \equiv \int_{0}^{\infty} d y \frac{y^{\alpha}\left(1+r_{\lambda} y^{2}\right)^{\beta}}{\left(1+y^{2}\right)^{\gamma}\left(1+r_{\nu} y^{2}\right)^{\delta}}
$$

Thus, $A, B, C, E, F, G$ are functions of the variables, $d^{\prime}, r_{\nu}$, and $r_{\lambda}$.

Before we analyze the $R G$ recursion relations in detail, it is interesting to note that Eqs. (6) and (9) have the similar expressions. To make parameters unchanged, if we set $\partial r_{\nu} / \partial l=0$ and $\partial U_{\rho} / \partial l=0$, we can easily get the relation, $z-2=-\left(2-d^{\prime}+2 \rho\right) / 3$. So the dynamical exponent can be written as

$$
z=2-\frac{2}{3}\left(\rho-\frac{d^{\prime}-2}{2}\right), \quad \alpha=\frac{2}{3}\left(\rho-\frac{d^{\prime}-2}{2}\right),
$$


provided $\nu_{\perp} \neq 0$ and $U_{\rho} \neq 0$. Thus, if there exists a stable fixed point at finite values of $\nu_{\perp}$ and $U_{\rho}$, we then immediately have the expressions for $z$ and $\alpha$.

In order to find the stable fixed point of the recursion relations, it is of help to recall the RG flow for the case of the white noise, $\rho=0$ at $d^{\prime}=2$ [1]. For this case, there exist the fixed points $r_{\nu}^{*}= \pm r_{\lambda}$, which were obtained by setting $\partial r_{\nu} / \partial l=0$. For $r_{\nu}^{*}=r_{\lambda}$, the fixed point of the effective coupling constant, $U \equiv D \lambda^{2} / \nu^{3}$ at zero, is unstable, while it is stable for $r_{\nu}^{*}=-r_{\lambda}$. Thus for $r_{\lambda}<0$, the nonlinear terms of the AKPZ equation become irrelevant even though they are finite, and the AKPZ equation belongs to the EW universality class. Following the similar steps, we have analyzed the recursion relations for the case of correlated noise with $d^{\prime}=2$ first. When $d^{\prime}=2$, Eq. (7) becomes explicitly as

$$
\frac{\partial r_{\nu}}{\partial l}=\frac{\pi\left(r_{\nu}+r_{\lambda}\right)}{8\left(1+\sqrt{r_{\nu}}\right)^{2} r_{\nu}^{\frac{3}{2}}}\left[\left(U_{0}+U_{\rho}\right)\left(1+\sqrt{r_{\nu}}\right)^{2}\left(r_{\lambda}-r_{\nu}\right)+4 \rho U_{\rho}\left(r_{\lambda}+2 r_{\lambda} \sqrt{r_{\nu}}-2 r_{\nu}^{\frac{3}{2}}-r_{\nu}^{2}\right)\right] .
$$

Thus, $r_{\nu}^{*}=-r_{\lambda}$ is the solution of $\partial r_{\nu} / \partial l=0$ even for the case of correlated noise. Solving $\partial U_{0} / \partial l=0$ and $\partial U_{\rho} / \partial l=0$ with $r_{\nu}^{*}=-r_{\lambda}$ in Eqs. (8) and (9), we then find two fixed points other than that of $U_{0}^{*}=U_{\rho}^{*}=0$ which is unstable for $\rho>0$ anyway. The stability analysis shows that, of the two new fixed points, one is unstable and the other is stable for all $\rho>0$ and $r_{\lambda}<0$. The stable fixed point is located at

$$
\begin{gathered}
U_{\rho}^{*}=\frac{4 \rho \sqrt{r_{\nu}^{*}}}{\pi}\left(\frac{-\zeta-9+3 \sqrt{\zeta^{2}+2 \zeta+9}}{\zeta^{2}}\right), \\
U_{0}^{*}=\frac{4 \rho \sqrt{r_{\nu}^{*}}}{\pi}\left(\frac{\zeta^{2}+4 \zeta+9-(\zeta+3) \sqrt{\zeta^{2}+2 \zeta+9}}{\zeta^{2}}\right),
\end{gathered}
$$

in the one-loop approximation where

$$
\zeta=\frac{12 \rho \sqrt{r_{\nu}^{*}}}{\left(1+\sqrt{r_{\nu}^{*}}\right)^{2}}
$$

Thus, we indeed have a stable fixed point in the 'off-axis' region and hence the dynamic exponents are given by Eq. (11) with $d^{\prime}=2$. As $\rho$ decreases to zero, the stable fixed point moves to $\left(U_{0}^{*}, U_{\rho}^{*}\right)=(0,0)$, and the case of white noise is recovered. As mentioned before, the 
recursion relations for $\chi \neq 1$ could not be derived explicitly. Thus we consider the case of $\chi \neq 1$ by extrapolating the analysis for $\chi=1$ with the recursion relations, Eqs. (6-9) in the following way. Since $\chi \neq 1$ means $r_{\lambda}=0$, we examine the behavior of the fixed point by taking the limit $r_{\lambda} \rightarrow 0^{-}$for fixed $\rho$. As can be seen in Eq. (13) and (14), the stable fixed point moves to the origin in the parameter space as $r_{\lambda}$ approaches to $0^{-}$, which suggests that the case of $\chi \neq 1$ belongs to the weak coupling limit.

For general dimensions $d^{\prime}$, the explicit expressions of the recursion relations are more complicated. Thus, it is difficult to derive the analytic formula for $r_{\nu}^{*}$ from $\partial r_{\nu} / \partial l=0$. Instead, the stable fixed point, $\left(r_{\nu}^{*}, U_{0}^{*}, U_{\rho}^{*}\right)$, is found numerically by iterating the RG transformation. We find that the stable fixed point exists in the off-axis region of the parameter space for $d^{\prime}<d_{c}^{\prime} \equiv 2+2 \rho$, and it locates at $U_{0}^{*}=U_{\rho}^{*}=0$ for $d^{\prime}>d_{c}^{\prime}$. This is in accord with Eq. (9) which shows that the fixed point at $U_{0}^{*}=U_{\rho}^{*}=0$ is unstable for $2-d^{\prime}+2 \rho>0$. Fig. 1 shows a typical RG flow diagram projected onto the $\left(U_{\rho}, U_{0}\right)$ plane at $d^{\prime}=2.5$. The existence of the off-axis fixed point implies that the nontrivial exponents $z$ and $\alpha$ are given by Eq. (11) for $d<d_{c}^{\prime}$.

The analytic formula, Eq. (11) is derived from the one-loop approximation RG transformation. However, it is in fact exact to all orders. The invariance of the noise spectrum $D(k)$ under the scale change, $x \rightarrow b x, t \rightarrow b^{z} t$, and $h \rightarrow b^{\alpha} h$ gives the exact exponent identity $2 \rho-d^{\prime}-2 \alpha+z=0$ as argued in [3]. This together with the relation $\alpha+z=2$ gives Eq. (11). The difference between the case treated in [3] and in this work is the range of applicability of Eq. (11). For the isotropic case, the formula is valid for $d^{\prime}<3 / 2$ and $\rho_{\min }<\rho<\rho_{\max }$ with approximately known $\rho_{\max }$ and $\rho_{\min }$. However, for the anisotropic case, the formula for the dynamic exponent is valid for $d^{\prime}<d_{c}^{\prime}=2+2 \rho$ and $\rho>0$. Hence the dynamic exponent $z$ in $d^{\prime}=2$ is nontrivial for finite $\rho$. Since the exponents $\alpha$ and $z$ are nontrivial in $d^{\prime}=2$, it would be interesting to introduce a stochastic model associated with the AKPZ equation having the alternative signs of $\lambda$ 's for correlated noise. A possible candidate for such a stochastic model would be a generalization of the $2+1$ dimensional Toom 
model introduced for the white noise [8] by replacing the random deposition by the spatially correlated deposition following the Lévy flight distribution. This conjecture is based on the analogy to the isotropic case, where the correlated noise was simulated by the correlated particle deposition following the Lévy flight distribution [10]. The simulations of the model is underway [1].

In summary, we have considered the anisotropic KPZ equation with the spatially correlated noise, using the one-loop dynamic RG transformation. In this study, the case that the signs of the nonlinear terms are opposite is interesting for both of white and correlated noises. Unlike the case of white noise, the stable fixed point exists in the "off-axis" region of the parameter space of the effective coupling constants $U_{0}$ and $U_{\rho}$ if $d^{\prime}<d_{c}^{\prime}=2+2 \rho$ for the case of correlated noise. From the stable fixed point, the dynamic exponent $z$ is determined as $z=2-\frac{2}{3}\left(\rho-\frac{d^{\prime}-2}{2}\right)$ and the roughening exponent $\alpha=2-z$. Therefore, the nontrivial values of the exponents $\alpha, z$ are present in $d^{\prime}=2$ for finite $\rho$. For $d^{\prime}>d_{c}^{\prime}$, the stable fixed point is located at the origin in the parameter space, and the AKPZ equation belongs to the Edwards-Wilkinson's universality class.

This work is supported in part by the KOSEF under the contract No 941-0200-006-2 and through the SRC program of SNU-CTP, and in part by the BSRI program of Ministry of Education, Korea. 


\section{REFERENCES}

[1] For a recent review, see, for example, F. Family and T. Vicsek, Dynamics of Fractal Surfaces (World Scientific, Singapore, 1991).

[2] M. Kardar, G. Parisi and Y. Zhang, Phys. Rev. Lett. 56, 889 (1986).

[3] E. Medina, T. Hwa, M. Kardar, and Y. C. Zhang, Phys. Rev. A 39, 3053 (1989).

[4] D. E. Wolf, Phys. Rev. Lett. 67, 1783 (1991).

[5] R. Cuerno and A.-L. Barabási, (preprint).

[6] S. Clarke, M. R. Wilby, and D. D. Vvedensky, Surf. Sci. 255, 91 (1991); D. D. Vvedensky, A. Zangwill, C. N. Luse, and M. R. Wilby, Phys. Rev. E 48, 852 (1993).

[7] S. F. Edwards and D. R. Wilkinson, Proc. R. Soc. London A 381, 17 (1982).

[8] H. Jeong, B. Kahng, and D. Kim, Phys. Rev. Lett. 67, 747 (1993); J. Korean Phys. Soc. 27, 124 (1994).

[9] B. Derrida, J. L. Lebowitz, E. R. Speer, and H. Spohn, Phys. Rev. Lett. 67, 165 (1991); J. Phys. A 24, 4805 (1991); A. L. Toom, in Multicomponent Random Systems, edited by R. L. Dobrushin and Ya. G. Sinai (Marcel Dekker, New York, 1980).

[10] P. Meakin and R. Jullien, Europhys. Lett. 9, 71 (1989); Phys. Rev. A 41, 983 (1990).

[11] H. Jeong, B. Kahng, and D. Kim, (unpublished). 


\section{FIGURES}

FIG. 1. Plot of the flow diagram of the RG transformation in the parameter space of $U_{\rho}$ and $U_{0}$ for $d^{\prime}=2.5, \rho=0.5$ and $r_{\lambda}=-2.5$. 